\title{
Labeling, Instructions for Use or Training Problem
}

National Cancer Institute

\section{Source}

National Cancer Institute. Labeling, Instructions for Use or Training Problem. NCI

Thesaurus. Code C63072.

Problem associated with device markings/labeling, instructions for use, training and maintenance documentation or guidelines. 